\title{
Determining heart parameters through left ventricular automatic segmentation for heart disease diagnosis
}

\author{
Antonio Fernández-Caballero ${ }^{\mathrm{a}, \mathrm{b}, *}$, José M. Vega-Riesco ${ }^{\mathrm{a}}$ \\ ${ }^{a}$ Instituto de Investigación en Informática de Albacete (I3A), Universidad de Castilla-La Mancha, 02071 Albacete, Spain \\ ${ }^{\mathrm{b}}$ Departamento de Sistemas Informáticos, Escuela Politécnica Superior de Albacete, Universidad de Castilla-La Mancha, 02071 Albacete, Spain
}

\begin{abstract}
Segmentation of the left ventricle in echocardiographic images is a task with important diagnostic power. This paper describes a method for the calculation of the ventricle's contours in long- and short-axis views. The segmentation process starts with a complex border detection process, followed by the application of a generalized Hough transform to detect curves and ended up through an active-contour algorithm. Afterwards, parameters left ventricular area in a short-axis view, left ventricular length in a long-axis view, left ventricular volume, left ventricular mass, and heart-wall thickness are specifically calculated.
\end{abstract}

(C) 2007 Elsevier Ltd. All rights reserved.

Keywords: Echocardiography; Left ventricle; Cardiological parameters; Segmentation; Active-contour

\section{Introduction}

Cardiovascular diseases are a major health concern worldwide. Of all the known heart diseases, hypertension, coronary heart disease, rheumatic valvular heart disease, chronic cor pulmonale, and congenital heart disease have been identified as the five most important (Fuster, Alexander, \& O'Rourke, 2001; Yan, Jiang, Zheng, Peng, \& Li, 2006). The left ventricle and in particular the endocardium is a structure of particular interest since it performs the task of pumping oxygenated blood to the entire body (Paragios, Jolly, Taron, \& Ramaraj, 2005). Therefore, segmentation of the left ventricle in echocardiographic images is a task with important diagnostic power. More concretely, contour extraction is an important criterion for subjective evaluation of the cardiac function and has become an area of focus (Mishra, Dutta, \& Ghosh, 2003). This paper describes a method for obtaining a series of parameters from the heart's left ventricle which are useful in the detec-

\footnotetext{
* Corresponding author. Address: Instituto de Investigación en Informática de Albacete (I3A), Universidad de Castilla-La Mancha, 02071 Albacete, Spain. Tel.: +34 967 599200; fax: +34 967599224.

E-mail address: caballer@dsi.uclm.es (A. Fernández-Caballero).
}

tion of certain heart diseases based on echocardiographic images. The bulk of the article describes methods for left ventricle segmentation in short- and long-axis views to calculate cardiac parameters useful for medical personnel. The following parameters are specifically calculated:

- Left ventricular area in a short-axis view.

- Left ventricular length in a long-axis view.

- Left ventricular volume.

- Left ventricular mass.

- Heart-wall thickness.

To solve these problems a set of algorithms providing left ventricular segmentation will be used. The problem that physicians face with echocardiographies is its great uncertainty. Each doctor decides where to place the cursor to take the measurements (Braunwald, Zipes, Libby, \& Bonow, 2004). If he or she is not a well-qualified expert in this type of measurements, he can make considerable mistakes. This paper is, thus, addressed to the elimination of the observer's uncertainty. This is achieved by automating the whole segmentation process, as well as the calculation of the results. We aim to carry out an approximation to the ventricle's contours in a long- and short-axis views 
through an active-contour algorithm. These algorithms are efficient at finding object edges, in this case, from the left ventricle. The trouble with the algorithm, though, is that the initial points must be introduced manually so that the algorithm approximates the object in question. This is solved with a Hough transform, a technique used to find objects with certain shapes. Therefore, when the transform finds an object in the image in the shape of a left ventricle, this information will be passed on to the active-contours algorithm to approximate and perfect the resolution. It is then possible to calculate the necessary parameters.

The aim of this approach is to automate these calculations, especially the detection of the left ventricle in a long-axis view, which is the one the doctors use most. No bibliographical reference has been found in the state-ofthe-art study for automatic ventricle detection in this view. Therefore, we regard this as an important contribution of the present paper. We do not expect to get perfect calculations, since this would require more sophisticated filters to process the images (Nastar \& Ayache, 1993), and this is not the aim of this paper. However, the results obtained are very close to reality, as it will be shown throughout this article.

\section{Antecedents in left ventricle segmentation}

This section will include some prior studies carried out in the field of echocardiographic images, specifically left ventricle segmentation starting from contour extraction models. There are many published works in the matter (Buda et al., 1983; Mikic, Krucinski, \& Thomas, 1998; Pardo, Solé, \& Cabello, 2000; Pincum, Schwartz, Corday, Fujibayashi, \& Meerbaum, 1986). We will list the most relevant publications related to this paper.

The methods suggested by Chu, Delp, and Buda (1988) require mostly the grey-level information along with some user-defined initial contours to extract the boundary in the images. Nastar and Ayache (1993) have proposed a basic model for the contour as a set of mass linked by springs to track the motion in a sequence of 2D images. However, the method proposed by Staib and Duncan (1992) implemented a probabilistic deformable model considering the boundary as two-dimensional deformable object using maximum posteriori estimate. A more recent work by Chalana, Linker, Haynor, and Kim (1996) reports an interesting approach to detect epicardial and endocardial boundaries of short-axis echocardiographic sequences using a multiple-active-contour model, an extension to the original model proposed by Kass, Witkin, and Terzopoulos (1988). The multiple-active-contour model is a special case of active surface model where the surface is represented as a sequence of planar contours. The algorithm requires user-defined initial approximation for epicardial boundary that detects the contour by computing gradients using Canny's edge detection method. The variance of the Gaussian kernel used to convolve the gradient image progressively decreases to intensify the convergence.
The optimized contours of the epicardial boarders are used as initial approximation for endocardial boundary with empirically determined values of the snake model parameters. A similar automated contour extraction algorithm proposed by Ranganath (1995) is applicable to spin and gradient echo magnetic resonance imaging (MRI) image sequences. It suggests a contour propagation technique to track the boundary in a sequence despite its poor temporal resolution. In Malassiotis and Strintzis (1999), a temporal learning-filtering procedure is applied to perfect the left ventricular (LV) boundary detected by an active-contour model. Instead of making prior assumptions about the LV shape or its motion, the information increases directly from the images and it is exploited to achieve a more coherent segmentation. A Hough transform technique is used to find an initial approximation of the object boundary at the first frame of the sequence. Then, an active-contour model is used in a coarse-to-fine framework, for the estimation of a noisy LV boundary.

More recently, automatic detection of the boundary of left ventricle in a sequence of cardiac images has been proposed (Mishra et al., 2003). The contour detection algorithm is formulated as a constrained optimization problem based on active-contour model. The optimization problem has been solved using a genetic algorithm (GA). The result obtained by the proposed GA based approach is compared with conventional nonlinear programming methods. In Montagnat, Sermesant, Delingette, Malandain, and Ayache (2003), a 4D (3D + time) echocardiographic image anisotropic filtering and a 3D model-based segmentation system are presented. To improve the extraction of left ventricle boundaries, the authors rely on two pre-processing stages. First, they apply an anisotropic filter that reduces image noise. This $4 \mathrm{D}$ filter takes into account the spatial and temporal nature of echocardiographic images. Second, they adapt the usual gradient filter estimation to the cylindrical geometry of the 3D ultrasound images. The reconstruction of the endocardium takes place by deforming a deformable simplex mesh having an a priori knowledge of left ventricle shape and that is guided by a region-based data attraction force. In another approach (Shekhar, Zagrodsky, \& Walimbe, 2004), images of the left ventricle are acquired both at rest and upon stress; these are then compared to detect the onset of new wall motion abnormalities post-stress or worsening of existing wall motion abnormalities at rest. In a work (Angelini, Homma, Pearson, Holmes, \& Laine, 2005), a clinical study for segmentation of right and left ventricular volumes is introduced. A preprocessing of the volumetric data sets is performed using spatiotemporal brushlet denoising. Two deformable-model segmentation methods are implemented in $2 \mathrm{D}$ using a parametric formulation and in 3D using an implicit formulation with a level set implementation for the extraction of endocardial surfaces on denoised data.

This paper opens a door towards left ventricular detection in a long-axis view (two- or four-chambers). Although digital techniques have improved, the echocardiographic 
images are inherently noisy due to the air in the lungs; therefore, initial point detection relying on thresholds can be complicated, since these thresholds can change from one image to the next.

\section{Echocardiographic parameters for coronary disease detection}

\subsection{Two-dimensional echocardiography principals}

The term "echocardiography" refers to a group of tests where the ultrasound is used to examine the heart and record information in the form of echoes (sound wave reflections). The maximum limit for a sound to be audible is 20,000 cycles/s or $20 \mathrm{KHz}$. The frequency used in ultrasounds is from 1 to 10 million cycles/s. The frequencies used for adults are usually $2.0-5.0 \mathrm{MHz}$, whereas the ones used for children are usually higher, from 3.5 to $10 \mathrm{MHz}$. The resolution of the image registration, which is the ability to distinguish two objects near in space, is directly proportional to the frequency and inversely proportional to the length of the wave. A high frequency ultrasound (short wave) identifies objects less than $1 \mathrm{~mm}$ away. Rays with the lowest frequency and longest wave have a more deficient resolution. On the other hand, the intensity of sound penetration, which is the ability to transmit enough ultrasonic energy into the thorax to provide an adequate range, is inversely proportional to the signal's frequency. A high frequency ultrasonic ray (e.g. 5 or $10 \mathrm{MHz}$ ) does not penetrate a thick thoracic wall, thus lower frequency ultrasound rays are used for adults. Although this enables the rays to penetrate the thoracic wall, it partially sacrifices the resolution; however, even with a transducer that produces $2.5 \mathrm{MHz}$ rays, which is frequently used in adult echocardiographies, it is still possible to identify objects at 1 and $2 \mathrm{~mm}$ away from each other.

Ultrasonic rays move in a sector in such a way that a projection of the heart in the shape of a piece of cake is obtained. In most two-dimensional commercial echocardiographs, the ultrasonic rays move in such a way that approximately 30 slices/s are obtained. The ultrasonic ray can move mechanically oscillating a single transducer or rotating a series of transducers. Similarly, the ultrasound is electronically guided using the principles of phase sequences where several ultrasonic elements are used to form the ray and in which the elements' shot sequence is controlled. It is necessary to use a computer or a microprocessor to control the elements' shots and the direction of the ray.

A heart ultrasonic test is, essentially, an ultrasound anatomic incision where a beam of thin ultrasounds makes incisions in different places, depending on where the transducer or another source of ultrasonic rays is placed. Due to the increasing use of the different positions of the transducer, the American Society of Echocardiography has standardized the nomenclature used for the different posi- tions naming them supraesternal, paraesternal, apical and subcostal (Braunwald et al., 2004).

It is possible to obtain an infinite number of incisions from the heart for each of the above-mentioned positions. There are many heart tests and views reported in the literature. The American Society of Echocardiography has standardized the two-dimensional tests described (Braunwald et al., 2004). The society believed that all views could be categorized in three orthogonal planes. These planes are a plane over the long or major axis, a plane over the short or minor axis and the four-chambered (or cavities) plane. These planes can be obtained from more than one position of the transducer. The society recommends any examination within $45^{\circ}$ of a basic plane to be identified with the name of that plane. Therefore, there are multiple views within each plane. The angular modifications depend on the exact cardiac structure to be examined.

The long-axis view can be obtained by placing with the transducer in the apical, paraesternal (left sternal border) positions or in the supraesternal cavity. The short-axis view crosses the heart so that the left ventricle looks like a circle. The right ventricle wraps around the left ventricle. These views are obtained by placing the transducer in a paraesternal or subcostal position. The four-chamber view enables us to examine the four-chambers of the heart simultaneously and it is obtained by placing the transducer over the heart or in a subcostal position.

\subsection{Cardiac parameters}

Medical images obtained from two-dimensional echocardiographies will be used to calculate left ventricular area, length, volume and mass. With these data, we can infer the type of heart disease that the individual being studied may suffer from. Cardiac function assessment is a difficult but extremely important task for the study of patients with an evident or suspected heart disorder. Ventricular functioning is related to the simple pumping function of the ventricle revealed as cardiac expense or output and expressed per heart beat or per minute. Ventricular function relates these indicators of ventricular performance with certain preloaded measurements, such as volume, telediastolic dimension and pressure or wall strain.

The angiographic methods are the most widely used for measuring heart chamber volume and also ventricular wall thickness. It is also possible to use techniques which do not involve corporal penetration to assess ventricular volume or dimensions. In this project, we will use images taken without corporal penetration, such as two-dimensional echocardiographies.

Methods that use cardiac catheterization and quantitative coronary angiography to study the function and contractibility of the heart are the most exact. However, they are not without risk or discomfort for patients, due to corporal penetration. We will, therefore, use less bloody medical images (two-dimensional echocardiographs) obtaining similar results. 


\subsubsection{Left ventricular volume}

The most widely used method to calculate left ventricular volume is the area-length method developed by Dodge (Braunwald et al., 2004). Left ventricular volume is calculated by using the volume formula for an ellipsoid, since volumes calculated this way (and checked through a regression equation) are correlated with ventricular volume directly measured. Left ventricular long-axis $(L)$ is directly measured; that is to say, the distance between the base point and the aortic valve. Afterwards, left ventricular diameter $(D)$ is calculated using the formula:

$D=4 \cdot \frac{A}{L}$

where $A$ is the area of left ventricular cavity in $\mathrm{cm}^{2}$.

The left ventricle is usually in the shape of an ellipsoid of revolution with a larger and two smaller diameters. Based on this, left ventricular volume is calculated using the formula:

$V=\frac{4}{3} \cdot \pi \cdot \frac{L}{2} \cdot \frac{D_{\mathrm{AP}}}{2} \cdot \frac{D_{\mathrm{lat}}}{2}$

where $V$ is the volume in $\mathrm{ml}, L$ is the long-axis in $\mathrm{cm}$ in the $\mathrm{AP}$ (anteroposterior) or lateral (planimetry), $D_{\mathrm{AP}}$, and $D_{\text {lat }}$ is the diameter (short-axes) in $\mathrm{cm}$, calculated for AP and lateral projections, respectively. At the same time, these diameters are calculated based on the formula for the area of an ellipse $(A)$ as follows:

$D=\frac{4 \cdot A}{\pi \cdot L}$

Real ventricular volume is determined from the volume calculated by using a regression formula, which takes into account the volume taken up by the papillary muscles and the chordae tendinae inside the left ventricular cavity. The exactness of this method has been investigated in human hearts obtained by necropsy, and in models and moulds taken from these hearts. A high correlation was noted.

Under normal conditions, left ventricular end-diastolic volume (SD, stroke diastolic) is, on the average, $70 \pm$ $20 \mathrm{ml} / \mathrm{m}^{2}$. In general, left ventricular function decreases when the ventricular end-diastolic volume is clearly high (that is $>110 \mathrm{ml} / \mathrm{m}^{2}$ or $>2$ standard deviation above the normal average) and total stroke volume or cardiac output and index have decreased or are within the normal limits, as long as both heart rate and blood pressure are normal.

Left ventricular stroke volume (SV) is calculated by taking the difference between the end-diastolic volume (EDV) and the end-systolic volume (ESV). Ejection fraction (EF) is the coefficient of SV divided by EDV:

$\mathrm{EF}=\frac{\mathrm{SV}}{\mathrm{EDV}}$

A two-dimensional echocardiography is user-friendly for calculating left ventricular end-diastolic volume (EDV). According to the method,
$\mathrm{EDV}=\left(D_{\max } \cdot \frac{L_{\max }}{4} \cdot 4.35\right)-6.44$

where $D_{\max }$ is the longest minor diameter determined by the paraesternal long-axis and four- and two-chamber views, and $L_{\max }$ is the greatest long-axis obtained in the apex projections.

\subsubsection{Left ventricular mass}

This is defined as the difference between the total ventricular volume (calculated based on the product of the left ventricular myocardial length and the short-axis left ventricular area) and the left ventricular chamber volume. This method has been validated with regard to the real ventricular mass. Therefore, the product of the volume of the wall multiplied by the heart muscle density (1.050) is equal to the mass:

$$
\begin{aligned}
M= & \left(\left(\frac{4}{3} \cdot \pi \cdot \frac{L+2 h}{2} \cdot \frac{D_{\mathrm{AP}}+2 h}{2} \cdot \frac{D_{\mathrm{lat}}+2 h}{2}\right)+V\right) \\
& \cdot 1.05
\end{aligned}
$$

where $h$ is the left ventricular wall thickening in $\mathrm{cm}, 1.050$ is the heart muscle density, $D_{\mathrm{AP}}$ and $D_{\text {lat }}$ are the left ventricular diameters in $\mathrm{cm}$ in the anteroposterior and lateral views, respectively, and $V$ is the left ventricular volume in $\mathrm{ml}$.

When single-plane methods are applied, we assume that $D_{\mathrm{AP}}=D_{\text {lat }}$,

$M=\left(\left(\frac{\pi}{6} \cdot(L+2 h) \cdot \frac{4 \cdot A}{\pi \cdot L}+2 h^{2}\right)+V\right) \cdot 1.05$

where $A$ is the area of the silhouette of a single-plane.

Left ventricular wall thickening usually is an average of $10.9 \pm 2.0 \mathrm{~mm}(\mathrm{SD})$; left ventricular mass, $92 \pm 16 \mathrm{~g} / \mathrm{m}^{2}$. A chronic heart dilation secondary to volume overload or to a primary myocardiopathy increases left ventricular mass the same way a chronic pressure overload does.

\subsubsection{Ejection fraction and myocardium fractional shortening}

The ejection fraction is the quotient of the stroke volume (SV) divided by the end-diastolic volume (EDV). It represents a global index of the degree of ventricular fiber shortening and, according to numerous empirical studies, it is regarded as a useful measure of the left ventricular pump function. On average, the left ventricular ejection fraction is $0.67 \pm 0.08$ (SD) for normal subjects. The ejection fraction varies in inverse function to the cardiac frequency.

The ejection fraction is directly related to the minor axis percentage shortening during systole. It is the basis for calculating the ejection fraction through echocardiographies. In general, the perpendicular diameter to the mid-point of the major axis is used, and the fractional shortening (FS) is calculated as follows and expressed in percentages:

$\mathrm{FS}=\frac{\text { telesystolic dimension }}{\text { telediastolic dimension }}$ 
3.2.4. Relationship between parameters and coronary disease

Once the parameters to be calculated are defined, let us take a look at how, using Table 1, we can find out the kind of disease the individual being studied has, by calculating left ventricular volume, mass and ejection fraction (Braunwald et al., 2004).

In Table $1, \mathrm{AS}$ is the aortic valve stenosis with maximum systolic gradient $>30 \mathrm{~mm} \mathrm{Hg}, \mathrm{AR}$ is the aortic regurgitation $>30 \mathrm{ml}$ per stroke, MS is the mitral stenosis with a valve area $<1.5 \mathrm{~cm}^{2}$, MR is the mitral regurgitation $>20 \mathrm{ml}$ per stroke, $A$ and $M$ are the combined aortic and mitral myocardiopathies. Myocardiopathy stands for acute or subacute myocardiopathy with coronary arteriosclerosis.

\section{Left ventricular segmentation in the short- and long-axis views}

Medical image segmentation is a difficult task due to low signal-to-noise ratios and poor contrast. In this project, we try to solve certain problems with the help of active-contours and other segmentation techniques, which will be applied to the images to process them and obtain better results. The application in medicine of image analysis and of computational vision and calculus has been a deciding factor in the increase of medical diagnosis accuracy and treatment plans. Segmentation is essential to any image analysis system.

Active-contour techniques allow to integrate different information elements into the segmentation process. It is possible to combine information from regions and boundaries with shape and/or statistical models. However, several problems must be solved in the classical implementation. One of them is requiring good initialization to stop the local minimum energy in the energy minimization process. A Hough transform will be used in the initialization process. The active-contours try to find object boundaries by heading for pixels where abrupt changes break the homogeneity of some of the scenes in the image. One of the most widely used features to identify these points is the gradient. High gradient is usually associated with a noticeable boundary. However, when dealing with medical images, it is very common to find boundaries with a gradient similar to that of the object boundary, due to noise or to other objects. Moreover, boundaries in the contours of interest might seem unconnected.
Left ventricle segmentation will be carried out as follows: first, the echocardiographic images will be preprocessed (both views), until gradient smoothness is obtained. After the images have been pre-processed, a Hough transform will be used to find the ventricle and, thus, obtain a better initialization of the active-contour. Once we have the pixels (snaxels) obtained by the transform, they are transferred to the active-contour algorithm, which completes the left ventricle segmentation. Finally, the parameters needed to get a diagnosis are calculated. A diagram of this process is shown in Fig. 1.

\subsection{Pre-processing: smoothing, gradient detection and gradient smoothing}

\subsubsection{Border detection: gradient}

Next, the processes followed to calculate the gradient image will be explained. The derivative from a continuous signal provides the local variations regarding the variable, so that the faster the variations, the greater the value of the derivative. Let us assume a two-dimensional function $f(x, y)$, the derivative is a vector which points in the direction of maximum variation of $f(x, y)$ and whose modulus is proportional to the said variation. This vector, known as $\nabla f(x, y)$, is called a gradient and is defined as

$$
\nabla f(x, y)=\left[\begin{array}{l}
\frac{\partial}{\partial x} f(x, y) \\
\frac{\partial}{\partial y} f(x, y)
\end{array}\right]=\left[\begin{array}{l}
f_{x}(x, y) \\
f_{y}(x, y)
\end{array}\right]
$$

Observing the previous equation, the gradient in a pixel $(x, y)$ is given by derivatives $f(x, y)$ along the axis of the orthogonal coordinates $x$ and $y$. The modulus and direction of the gradient are given by

$$
\begin{aligned}
& |\nabla f(x, y)|=\sqrt{f_{x}(x, y)^{2}+f_{y}(x, y)^{2}} \\
& \alpha(x, y)=\arctan \frac{f_{y}(x, y)}{f_{x}(x, y)}
\end{aligned}
$$

In the discrete two-dimensional case, the different approximations of the gradient operator are based on the differences in the image's grey-levels. For instance, the partial derivative $f_{x}(x, y)$ (row gradient) can approximate through the difference in adjacent pixels in the same row, that is to say,

\begin{tabular}{|c|c|c|c|c|c|}
\hline Group & Patients & Telediastolic vol. $\left(\mathrm{ml} / \mathrm{m}^{2}\right)$ & Stroke vol. $\left(\mathrm{ml} / \mathrm{m}^{2}\right)$ & $\operatorname{Mass}\left(\mathrm{g} / \mathrm{m}^{2}\right)$ & Ejection fraction \\
\hline Normal & - & $70 \pm 20.0$ & $45 \pm 13.0$ & $92 \pm 16.0$ & $0.67 \pm 0.08$ \\
\hline AS & 14 & $84 \pm 22.9$ & $44 \pm 10.1$ & $171 \pm 32.7$ & $0.56 \pm 0.17$ \\
\hline AR & 22 & $193 \pm 55.4$ & $92 \pm 30.0$ & $223 \pm 73.0$ & $0.57 \pm 0.13$ \\
\hline MS & 37 & $83 \pm 21.2$ & $43 \pm 11.9$ & $98 \pm 24.1$ & $0.57 \pm 0.14$ \\
\hline MR & 29 & $160 \pm 53.1$ & $87 \pm 21.3$ & $166 \pm 49.9$ & $0.47 \pm 0.10$ \\
\hline MS and MR & 29 & $106 \pm 34.4$ & $58 \pm 14.7$ & $119 \pm 27.8$ & $0.57 \pm 0.12$ \\
\hline
\end{tabular}

Table 1

Parameters versus diseases 


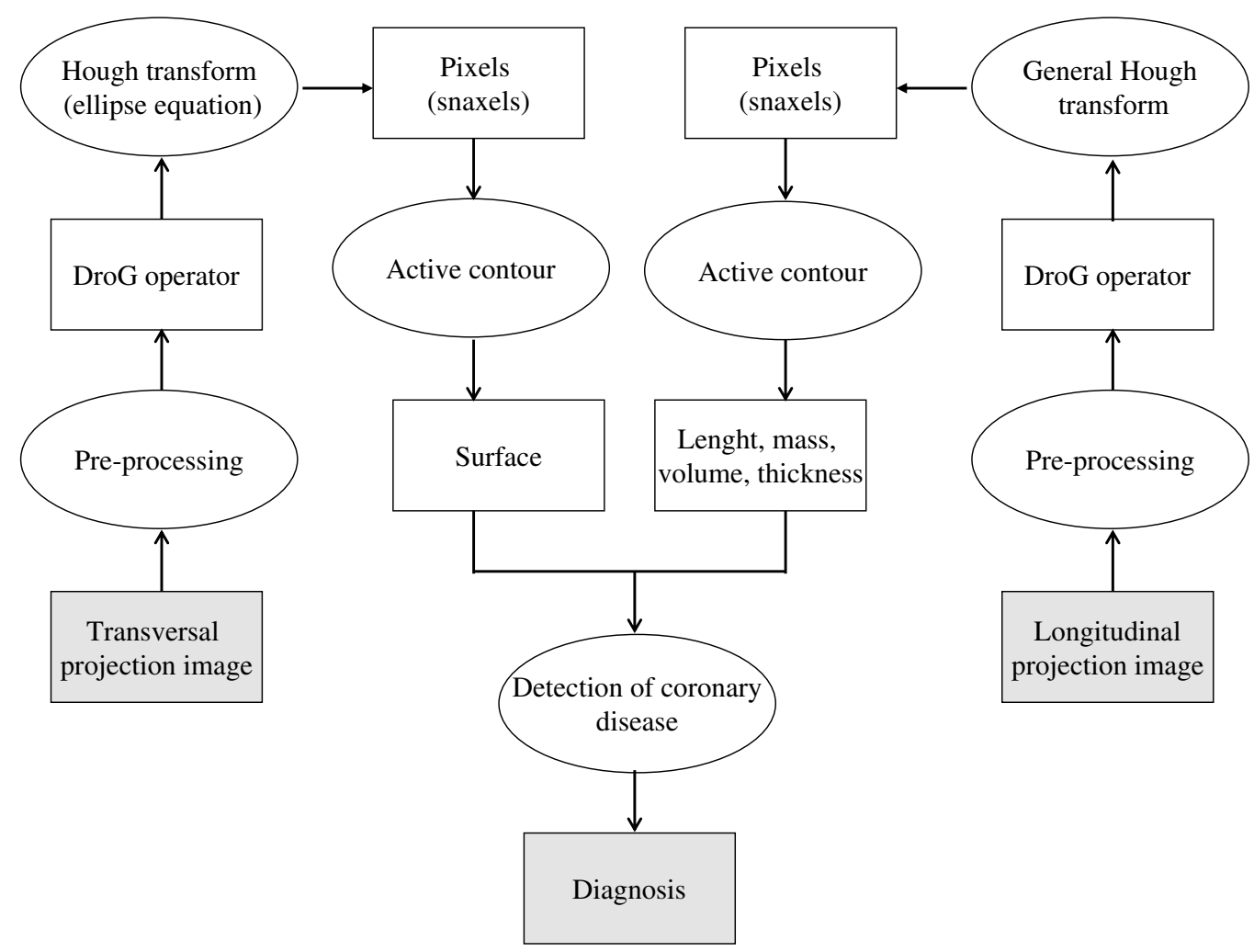

Fig. 1. Left ventricular segmentation in the short- and long-axis views for the detection of coronary diseases.

$f_{x}(x, y) \approx G_{F}(i, j)=\frac{F(i, j)-F(i, j-1)}{T}$

or also through the difference in separate pixels:

$f_{x}(x, y) \approx G_{F}(i, j)=\frac{F(i, j+1)-F(i, j-1)}{2 T}$

where it is assumed that, without the loss of generality, the row index $i$ grows from top to bottom and the column index $j$ from left to right. $\frac{1}{T}$ and $\frac{1}{2} T$ are scale factors. Row gradients $G_{F}$ and column gradients $G_{C}$ for each pixel are obtained through image convolution with a mask $H_{F}$ and $H_{C}$, respectively, that is to say

$$
\begin{aligned}
& G_{F}(i, j)=F(i, j) \otimes H_{F}(i, j) \\
& G_{C}(i, j)=F(i, j) \otimes H_{C}(i, j)
\end{aligned}
$$

where $H_{F}(i, j)$ and $H_{C}(i, j)$ are the impulse responses for the row and column gradients, respectively. The magnitude and orientation of the gradient vector are obtained according to the previous equations. Although these equations require great computational cost to calculate the magnitude, the following expression is normally used:

$$
\begin{aligned}
|G(i, j)| & =\sqrt{G_{F}(i, j)^{2}+G_{C}(i, j)^{2}} \\
& =\left|G_{F}(i, j)\right|+\left|G_{C}(i, j)\right|
\end{aligned}
$$

The Sobel operator is the operator used to calculate the gradient $\left(H_{F}\right.$ and $\left.H_{C}\right)$. This operator is more sensitive to diagonal edges and works somewhat better with medical images than the Prewitt operator, which is more sensitive to vertical and horizontal edges

$H_{F}(i, j)=\left[\begin{array}{lll}-1 & 0 & 1 \\ -2 & 0 & 2 \\ -1 & 0 & 1\end{array}\right]$

$H_{C}(i, j)=\left[\begin{array}{ccc}1 & 2 & 1 \\ 0 & 0 & 0 \\ -1 & -2 & -1\end{array}\right]$

The idea with this operator is to use an approximation of the gradient which is computationally efficient and more isotropic than other operators. The magnitude of directional derivative $g$ is defined as

$|g|=\frac{\text { intensity difference }}{\text { distance to neighbor }}$

The direction of $g$ is given by the unitary vector, from the central to the neighboring pixel. Gradient $G$ in the pixel taken is calculated from the sum of the directional derivatives with its eight neighbors, that is to say

$$
\begin{aligned}
G= & \left(\frac{c-e}{R}+\frac{e-g}{R}\right) \cdot \frac{(1,1)^{\mathrm{T}}}{R}+\left(\frac{a-e}{R}+\frac{e-i}{R}\right) \\
& \cdot \frac{(-1,1)^{\mathrm{T}}}{R}+\left(\frac{b-e}{R}+\frac{e-h}{R}\right) \cdot(0,1)^{\mathrm{T}} \\
& +\left(\frac{f-e}{R}+\frac{e-d}{R}\right) \cdot(1,0)^{\mathrm{T}}
\end{aligned}
$$


where $R=\sqrt{2}$. The operand in the previous expression results

$$
G=\left|\begin{array}{l}
2(f-d)+(c-g-a+i) \\
2(b-h)+(c-g+a-i)
\end{array}\right|
$$

Since the previous expression should scale with factor $\frac{1}{4}$ to obtain unity gain in the positive and negatives sides of the operator, the equation really provides a value 8 times greater than the real value. Fig. 2 shows the implementation of a border detection algorithm.

Once the gradient modulus is calculated for each pixel $G(i, j)$, a thresholding operation is usually applied, where it is decided which pixels belong and which do not belong to an edge of the image. Threshold choice is critical: a low threshold would detect fluctuations as edges due to noise (especially using echocardiographic images) and would give rise to multiple responses. On the contrary, a high threshold would not detect many of the edges in the image. For this threshold, we use the same grey-level usually the heart tissue has (see Fig. 3).

The direction of the gradient in a pixel in the image is that of grey-level maximum variation. That is to say, per-

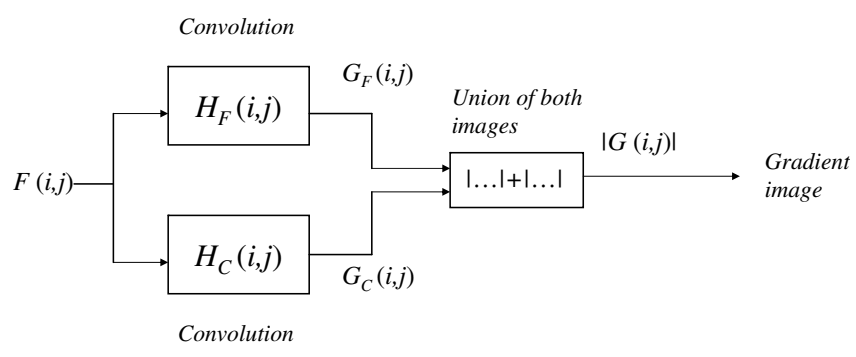

Fig. 2. Border detection algorithm. pendicular to the edge of the said pixel. This direction is not taken into account in the previous diagram, but it is very important because it supplies significant information for subsequent stages, since it will be used to apply a Hough transform. This transform will be used for image segmentation and to carry out a first approximation of the active-contour (see Fig. 4).

The size of the operator is of great importance with regard to detection and location. $2 \times 2$ and $3 \times 3$ operators are noise sensitive (especially the $2 \times 2$ ). This problem is usually solved by increasing the size of the mask which acts upon a greater number of pixels. This is similar to applying a pre-smoothing to the image and later applying the gradient operator.

\subsubsection{Smoothing}

Smoothing operations are primarily used for diminishing noise and/or spurious effects in an image as a consequence of the capturing process, digitalization, transmission or the very nature of the image. They are usually necessary prior to the application of an edge detector. The down side of using an image smoothing is that it implies edge diminution and blurring, and thus more difficulties in locating them. However, if a smoothing of the image was not applied prior to this, the noise would affect the gradient operator too much and would detect nonexisting objects. This would happen especially in echocardiographic images, since they are noisy by nature due to the air in patients' lungs.

For the smoothing, we will use a weighted average of the background, which takes into account the grey-level of the neighboring pixels that will be used. This type of smoothing, which assigns different weights to each pixel, is used to avoid blurring features in the image. A Gaussian filter will be used by means of the mathematical operations shown next:
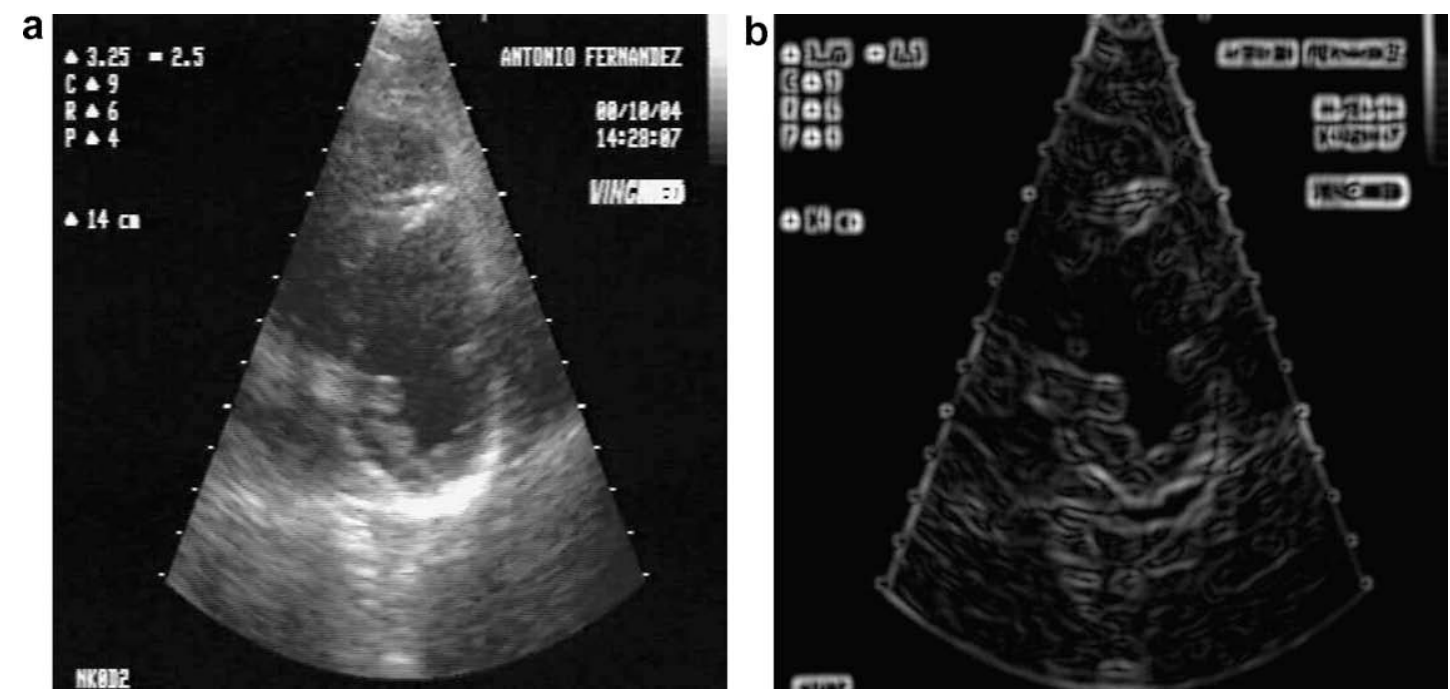

Fig. 3. Application of the gradient operator. (a) Original image of the left ventricle in the short-axis view. (b) Gradient image of the left ventricle in the short-axis view. 

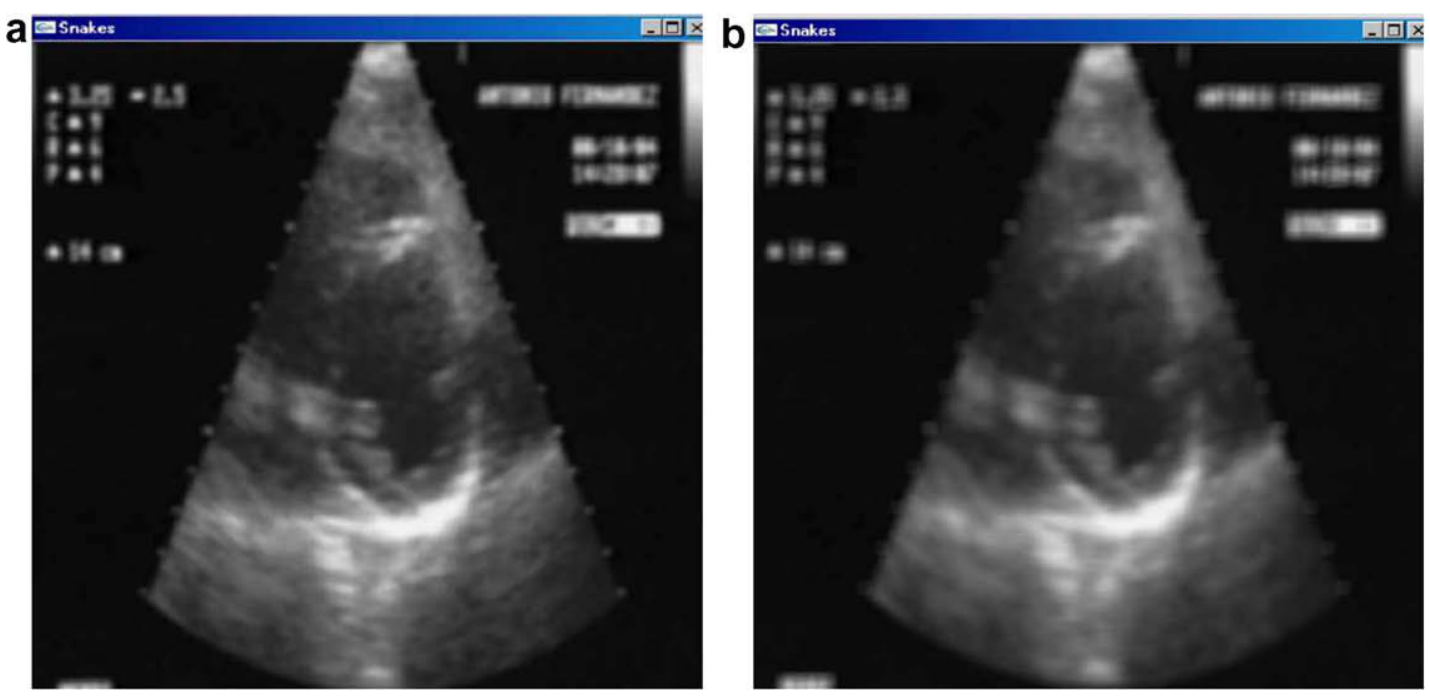

Fig. 4. Smoothing. (a) Image smoothed with $\sigma=7$. (b) Image smoothed with $\sigma=50$.

$g_{\sigma}(x, y)=\frac{1}{2 \pi \sigma^{2}} \mathrm{e}^{-\frac{\sqrt{x^{2}+y^{2}}}{2 \sigma^{2}}}=\frac{1}{2 \pi \sigma^{2}} \mathrm{e}^{-\frac{r}{2 \sigma^{2}}}=g_{\sigma}(r)$

with $r=\sqrt{x^{2}+y^{2}}$.

The convolution of function $g_{\sigma}(x, y)$ with a signal $f(x, y)$ gives rise to a new signal $h(x, y)$, where the value for each point is the result of working out the average, with different weights, of neighboring values at both sides of the said neighbors. Standard deviation $\sigma$ plays an important role in controlling the operator's degree of smoothing.

When we spoke earlier about the edge detection of an image, we said that threshold choice was critical. If it is too low, we could detect edges that do not belong to the object, due to noise, and if it is too high, we would not detect anything. Thresholding is not explicit; rather, when the snake acts, it does it over pixels that exceed a certain threshold (they attract the snake).

\subsubsection{DroG operator smoothing}

A very usual procedure to make the gradient operator less vulnerable to noise consists in previously smoothing the image. Both procedures (smoothing and gradient) can be combined into one through convolution:

$H(i, j)=H_{G}(i, j) \otimes H_{S}(i, j)$

where $H_{S}(i, j)$ is the original image smoothing and $H_{G}(i, j)$ is the gradient image. Gradient calculation for a continuous function $f(x, y)$ smoothed with $g_{\sigma}(x, y)$ is given by the composition of operations:

$\nabla\left[f(x, y) \otimes g_{\sigma}(x, y)\right]$

where $\nabla[\cdots]$ indicates the gradient operator. Since the gradient operator, as well as the convolution, is a linear operation, the previous equation can be expressed as

$$
\begin{aligned}
\nabla\left[f(x, y) \otimes g_{\sigma}(x, y)\right] & =f(x, y) \otimes \nabla\left[g_{\sigma}(x, y)\right] \\
& =f(x, y) \otimes \operatorname{DroG}(x, y)
\end{aligned}
$$

Operator DroG is a vector defined as

$$
\begin{aligned}
\operatorname{DroG}(x, y) & =\left|\begin{array}{l}
\frac{\partial}{\partial x}\left(g_{\sigma}(x) g_{\sigma}(y)\right) \\
\frac{\partial}{\partial y}\left(g_{\sigma}(x) g_{\sigma}(y)\right)
\end{array}\right|=\left|\begin{array}{l}
g_{\sigma}(y) \frac{\partial}{\partial x}\left(g_{\sigma}(x)\right) \\
g_{\sigma}(x) \frac{\partial}{\partial y}\left(g_{\sigma}(y)\right)
\end{array}\right| \\
& =\left[\begin{array}{l}
\frac{-x g_{\sigma}(x) g_{\sigma}(y)}{\sigma^{2}} \\
\frac{-y g_{\sigma}(x) g_{\sigma}(y)}{\sigma^{2}}
\end{array}\right]
\end{aligned}
$$

where $g_{\sigma}(x, y)=g_{\sigma}(x) g_{\sigma}(y)$.

To implement operator DroG into a digital image, it is necessary to discretize the components $x$ and $y$ in the previous equation. This discretization is carried out according to the value of standard deviation $\sigma$. Since echocardiographic images are very noisy, the smoothing is usually applied again over the imaged obtained with operator DroG (see Fig. 5).

\subsection{Hough transform}

A Hough transform is a tool which allows us to detect curves in an image. Although, at first, the curve has to be

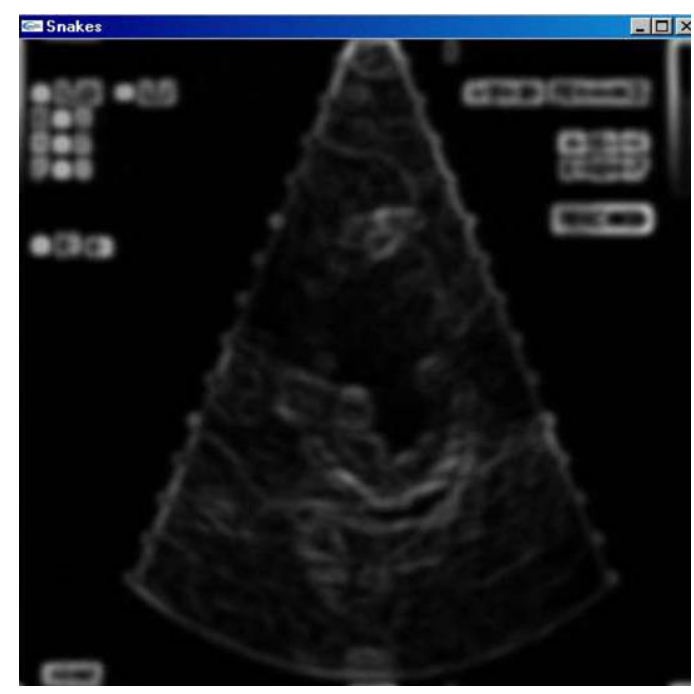

Fig. 5. Image obtained after DroG operator smoothing. 
specified by means of an analytical expression (the most common being the ones for straight lines, circumferences and ellipses), this technique is not all that restrictive, since it can be used to detect arbitrary curves, for which there is no equation. This will be verified in left ventricle detection in the long-axis. The main advantage of this technique is its efficiency with noisy images and with gaps in the object boundary. This is of great help in echocardiographic images, since they are known for having a lot of noise and not having completely joined contours.

When applying a Hough transform to an image, it is first necessary to obtain a binary image of the pixels which will probably be part of the boundary. In order to do this, the image smoothed with operator DroG is obtained from the original image. Then, a cut-threshold is applied. In the explanation of the Hough transform, the detection of circumferences will be used and later given general applicability to any equation. The detection of circumferences in an image is somewhat more complex than the detection of straight lines because circles have three parameters. The equation for a circumference is as follows:

$(x-a)^{2}+(y-b)^{2}=r^{2}$

where $a$ and $b$ are the center of the circumference and $r$ is the radius.

The aim is to find the different set of points that will satisfy the equation of the circumference for the different values of $a, b$ and $r$. To apply a Hough transform, it is necessary to discretize the parameter space into a series of, what is called, accumulator cells. This discretization is carried out for parameters $a, b$ and $r$. The accumulator will be three-dimensional in this case. That is to say and that it will have the following shape $A(a, b, r)$. The procedure consists in incrementing $a$ and $b$, determining $r$ through the previous equation and voting in the associated cell. The equation is then evaluated and at the end of the process, the number of votes obtained in each cell indicates the number of points that, except for discretization errors, satisfy the equation of the corresponding circumference. Therefore, cells with a greater accumulation of votes constitute the set of circumferences detected.

With the purpose of reducing the number of operations, when the length of the radius is known, local information about boundary location for each pixel can be used to obtain the position of the center of the circle. In practice, this is done by placing the center at a distance $R$ throughout the gradient direction for each pixel in the edge. This way, the number of points accumulated is equal to the number of edge pixels in the image, which implies great computational savings. To achieve this, the operator used for edge detection must be accurate enough (for example, the Sobel operator).

\subsubsection{Short-axis left ventricle detection from an ellipse}

Next, we will delve deeper into the problem. The shape of a left ventricle short-axis view is that of an "irregular" ellipse. However, we can approximate it to a regular ellipse, since having an equation that defines the ellipse makes it easier. To estimate left ventricular boundary in the shortaxis view through an ellipse, it is necessary to know the center of the ellipse, the size of the axes and the rotation angle. These data are estimated by using information about image intensity. Directly applying the Hough transform algorithm for ellipse detection implies searching in a fivedimensional space. Another alternative is to explore the information given by the gradient. The graph for an ellipse in polar coordinates is as follows:

$$
\begin{aligned}
& x=r_{1} \cos \phi \cos \theta+r_{2} \sin \phi \cos \theta+x_{0} \\
& y=-r_{1} \cos \phi \sin \theta+r_{2} \cos \phi \cos \theta+y_{0}
\end{aligned}
$$

where $r_{1}, r_{2}$ are the length of the axes, $\theta$ is the rotation angle, and $\left(x_{0}, y_{0}\right)$ is the center of the ellipse. By deriving the previous expressions with respect to $\phi$ we obtain

$$
\begin{aligned}
& \frac{\mathrm{d} x}{\mathrm{~d} \phi}=-r_{1} \sin \phi \cos \theta+r_{2} \cos \phi \sin \theta \\
& \frac{\mathrm{d} y}{\mathrm{~d} \phi}=r_{1} \sin \phi \sin \theta+r_{2} \cos \phi \cos \theta
\end{aligned}
$$

where $x_{0}, y_{0}$ have been eliminated. This way, parameters $r_{1}$, $r_{2}$ and $\theta$ are estimated, by using gradient direction for each pixel.

Next, we will show both algorithms used to apply a Hough transform:

$$
\begin{aligned}
& \forall \operatorname{pixel}(x, y) \text { belonging to the border } \\
& \forall A\left(r_{1}, r_{2}, \theta\right) \in S \\
& \text { solve } \min _{\phi}\left|\nabla G(x, y)\left[\frac{\mathrm{d} x}{\mathrm{~d} \phi}, \frac{\mathrm{d} y}{\mathrm{~d} \phi}\right]\right| \\
& \text { if }\left|\nabla G(x, y)\left[\frac{\mathrm{d} x}{\mathrm{~d} \phi}, \frac{\mathrm{d} y}{\mathrm{~d} \phi}\right]\right|<\varepsilon \text { then } V(A)=V(A)+|\nabla G(x, y)|
\end{aligned}
$$

where $G(x, y)$ is the gradient image solved with the Sobel operator, $S$ is the discretized space and $\varepsilon$ is the threshold for accumulator $V(A)$. The set of parameters which maximize $V(A)$ defines the ellipse or ellipses. Once this is over, we look for the greater value of $V(A)$, and that will be the center and the angle of the ellipse defined by the left ventricle.

Since we also have to use the left ventricle in a long-axis view, the same steps as those for the short-axis view must be taken. The problem here is that there is no longer an equation to approximate the ventricle; therefore, a generalized Hough transform has to be used. It is explained next (see Fig. 6).

\subsubsection{Generalized hough transform}

In the previous explanation about a Hough transform, it was possible to use analytical equations of a figure (circumference or ellipse) to transfer from an image coordinate space into a parameter space. However, since it is no longer possible to resort to equations which will define the desired 


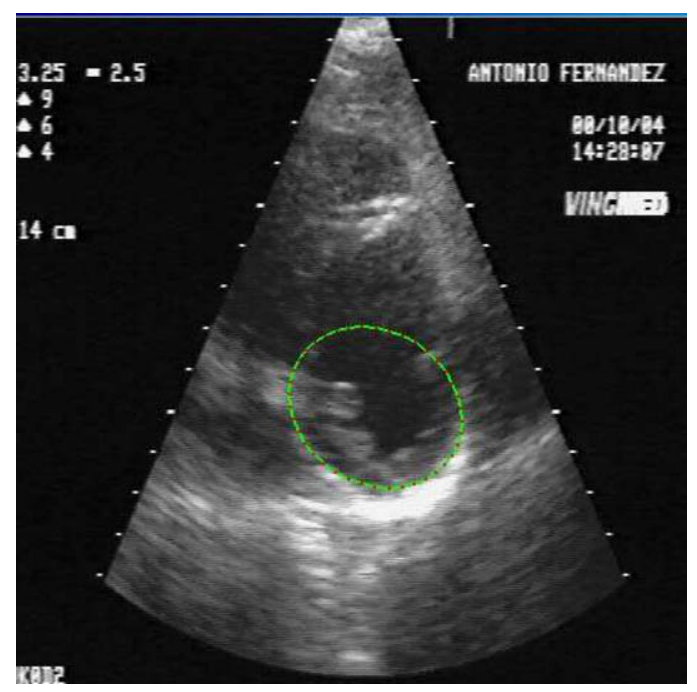

Fig. 6. Detection of the left ventricle (short-axis view).

figure, a generalized transform has to be used. The idea is to use a look-up table to define the existing relationship between the pixels in the figure and the Hough space parameters. The values in the look-up table have to be previously obtained using a figure prototype. We substitute the equation for the table with the chosen points. Assuming that the shape and direction of an object is known, the first step in the elaboration of the table is to choose a reference pixel at random $\left(x_{\text {ref }}, y_{\text {ref }}\right)$ in the object. From this point, the rest of the points will be defined and, thus, the figure.
The next step is to calculate direction $\beta_{i}$ and distance with reference point $r_{i}$ for each boundary point $\left(x_{i}, y_{i}\right)$.

Once the table has been elaborated, the Hough space is defined according to the possible positions of the figure in the image. When the Hough transform is applied, the number of cell coordinates to be increased, according to the expression, is calculated for every image pixel:

$$
\begin{aligned}
& x_{\text {ref }}=x+r \cos \beta \\
& y_{\text {ref }}=y+r \sin \beta
\end{aligned}
$$

where the values for $r$ and $\beta$ are obtained from the table.

We have assumed up to now that the orientation of the figure is known. If not, accumulator dimension would have to be increased (as in real situations) by incorporating a parameter $\phi$ which would consider the possible orientation of the object to be found. The previous expressions would change in the following way:

$$
\begin{aligned}
& x_{\text {ref }}=x+r \cos (\beta+\phi) \\
& y_{\text {ref }}=y+r \sin (\beta+\phi)
\end{aligned}
$$

for all discrete values considered by $\phi$.

Once the algorithm for a generalized Hough transform is proposed, we will explain, in the next point, how to locate the left ventricle in a long-axis view.

\subsubsection{Hough transform for left ventricle location (long-axis)}

The first step to construct this type of algorithm is to have the figure prototype to be found in the image (see Fig. 7a). Once we have the figure prototype, a reference

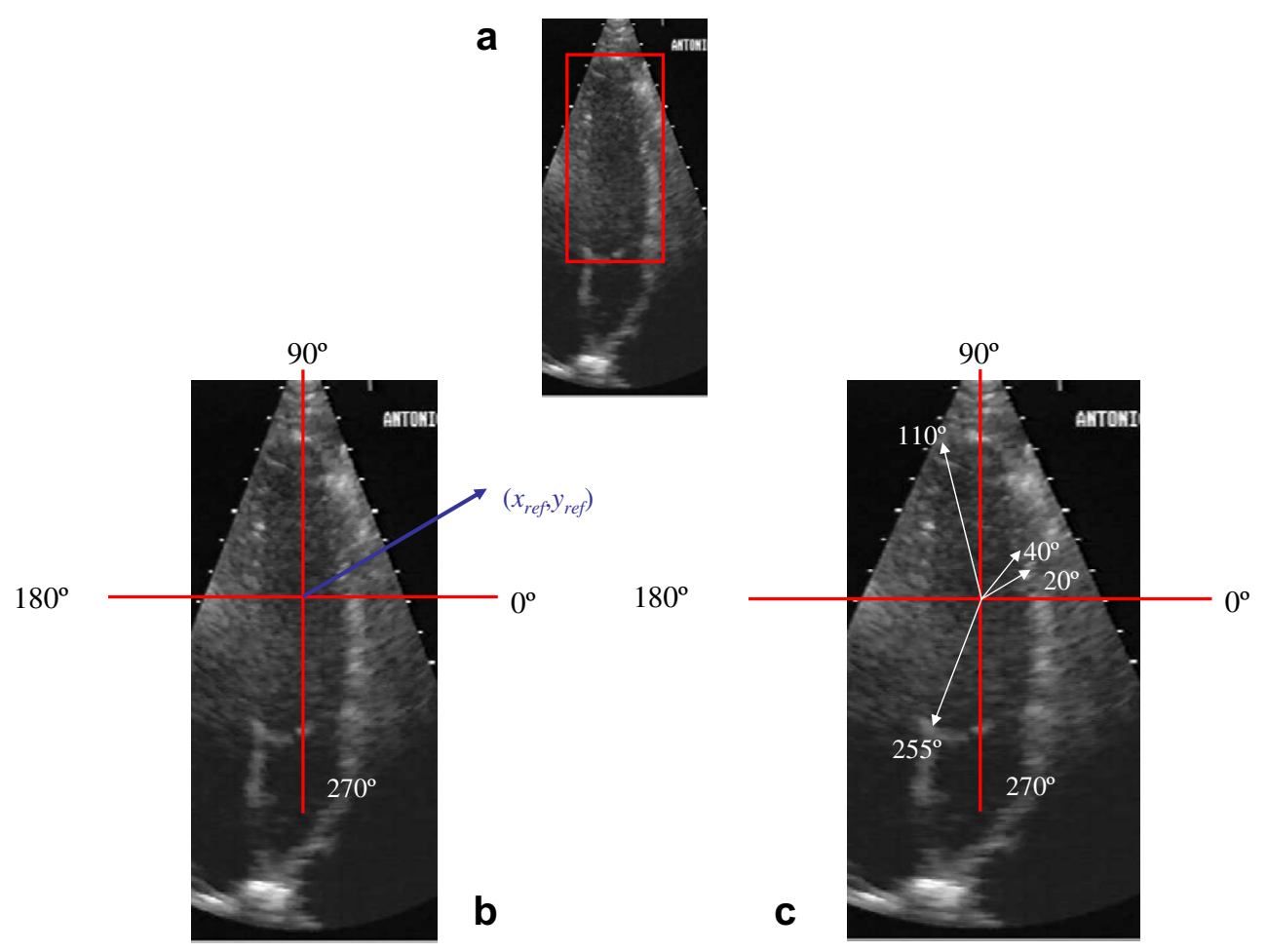

Fig. 7. (a) Left ventricle in the long-axis view. (b) Selection of points to define the left ventricle in the long-axis view. (c) Selection of points which defines the left ventricle. 
point is chosen $\left(x_{\text {ref }}, y_{\text {ref }}\right)$, from which the rest of the points that will make up the contour of the figure will be defined (see Fig. 7b). The central point in the image is usually chosen to ease the calculations. Once we have $\left(x_{\mathrm{ref}}, y_{\mathrm{ref}}\right)$, we can begin to construct the contour. The points which best display the characteristics of the figure are defined. The points will be defined in polar coordinates, that is to say, for their angle with regard to $\left(x_{\text {ref }}, y_{\text {ref }}\right)$ and their length $(L)$.

With these data and a simple trigonometric calculation, we can find the coordinates in $x$ and $y$

$x=L \cos \alpha+x_{\text {ref }}$

$y=L \sin \alpha+y_{\text {ref }}$

In Fig. 7c some points which have to be found have been defined. The idea is to define all points in $360^{\circ}$. Next, Table 2, which contains all points is shown.

This is the (look-up) table used as a reference for the location of the left ventricle. The relation between the image pixels and the Hough space parameters is defined this way. After applying the equations, the following coordinates (Table 3) are obtained. When representing these coordinates, the graphic shown as Fig. 8 is obtained.

Once we have the look-up table and have checked that it defines the left ventricle in a long-axis view, it is necessary to introduce more parameters to the equations. One of these parameters is information about the frequency of

Table 2

Points which define the left ventricle

\begin{tabular}{llllllll}
\hline$\alpha\left({ }^{\circ}\right)$ & $L$ & $\alpha\left({ }^{\circ}\right)$ & $L$ & $\alpha\left({ }^{\circ}\right)$ & $L$ & $\alpha\left({ }^{\circ}\right)$ & $L$ \\
\hline 0 & 13 & 103 & 37 & 210 & 15 & 280 & 35 \\
20 & 12 & 106 & 39 & 220 & 17 & 285 & 34 \\
40 & 14 & 110 & 28 & 230 & 20 & 290 & 33 \\
50 & 16 & 120 & 22 & 240 & 25 & 296 & 30 \\
60 & 19 & 130 & 18 & 245 & 30 & 300 & 25 \\
70 & 24 & 140 & 16 & 250 & 33 & 310 & 20 \\
80 & 32 & 150 & 15 & 255 & 36 & 320 & 17 \\
83 & 36 & 160 & 14 & 257 & 37 & 330 & 15 \\
87 & 39 & 170 & 13 & 260 & 37 & 340 & 14 \\
90 & 39 & 180 & 13 & 265 & 37 & 350 & 13 \\
96 & 39 & 190 & 13 & 270 & 37 & & \\
100 & 39 & 200 & 14 & 275 & 36 & & \\
\hline
\end{tabular}

Table 3

Coordinates of the left ventricle

\begin{tabular}{|c|c|c|c|c|c|c|c|}
\hline$x$ & $y$ & $x$ & $y$ & $x$ & $y$ & $x$ & $y$ \\
\hline 13.00 & 00.00 & -8.32 & 36.05 & -12.99 & -7.5 & 6.07 & -34.46 \\
\hline 11.27 & 4.10 & -9.37 & 32.68 & -13.02 & -10.92 & 8.79 & -32.84 \\
\hline 10.72 & 8.99 & -9.57 & 26.31 & -12.85 & -15.32 & 11.25 & -31.00 \\
\hline 10.28 & 12.25 & -11.00 & 19.05 & -12.50 & -21.65 & 13.15 & -26.96 \\
\hline 9.50 & 16.45 & -11.57 & 13.78 & -12.67 & -27.18 & 12.50 & -21.65 \\
\hline 8.20 & 22.55 & -12.25 & 10.28 & -11.28 & -31.00 & 12.85 & -15.32 \\
\hline 5.55 & 31.51 & -12.99 & 7.50 & -9.31 & -34.77 & 13.02 & -10.92 \\
\hline 4.38 & 35.73 & -13.15 & 4.78 & -8.32 & -36.05 & 12.99 & -7.50 \\
\hline 2.04 & 38.94 & -12.80 & 2.25 & -6.42 & -36.43 & 13.15 & -4.78 \\
\hline 0.00 & 39.00 & -13.00 & 0.00 & -3.22 & -36.85 & 12.80 & -2.25 \\
\hline-4.07 & 38.78 & -12.80 & -2.25 & 0.00 & -37.00 & & \\
\hline-6.77 & 38.40 & -13.15 & -4.78 & 3.13 & -35.88 & & \\
\hline
\end{tabular}

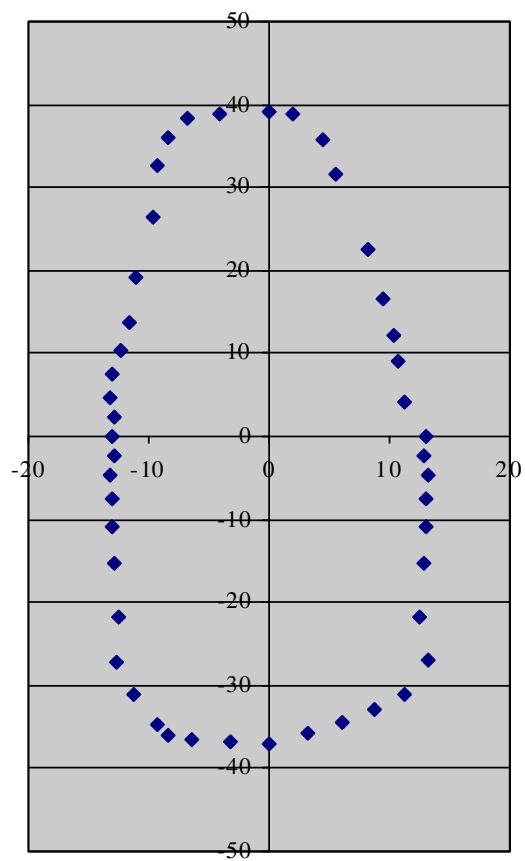

Fig. 8. Representation of the coordinates of Table 3.

the ultrasound, which enables us to know which scale factor the ultrasound is using. Depending on what it is, the ultrasound will make deeper slices. Its value appears in $\mathrm{cm}(14,16, \ldots)$. Depending on this value, the scale value for the objects that appear in the image will vary. After measuring the objects in the different depths used by the ultrasound, the following expression is obtained:

$\rho=\frac{\text { prof }}{139} \cdot \frac{1}{1.15}$

where prof is the depth the ultrasound uses. If this expression is added to the equations they would be as follows:

$x=(\rho \cdot L) \cos \alpha+x_{\text {ref }}$

$y=(\rho \cdot L) \sin \alpha+y_{\text {ref }}$

The next step is to realize that all ventricles are not the same, just like people who are not the same. Then, since the aim is to search for something in an image, we should 
bear in mind that the left ventricle can be of a different size, depending on whether it belongs to a man or a woman, the patient's age, scale factor, and so on. Taking this parameter into account, we have to introduce a new parameter to control the size of the ventricle during the search. This new parameter will be $\omega$

$x=(\omega \cdot \rho \cdot L) \cos \alpha+x_{\text {ref }}$

$y=(\omega \cdot \rho \cdot L) \sin \alpha+y_{\text {ref }}$

The results obtained with $\rho=1$ and $\omega=0.8$ are shown in Fig. 9a.

Lastly, one more parameter to control the ventricle's rotation angle has to be included. If this parameter were not included, it would only be possible to do a search with a certain angle. This is not true to reality, since the ventricle can have different positions. That is to say, the transducer controlled by the doctor. Parameter $\phi$ will be in charge of controlling the rotation angle. The equations will be modified as follows:

$x=(\omega \cdot \rho \cdot L)(\cos \alpha \cdot \cos \phi+\cos \alpha \cdot \sin \phi)+x_{\text {ref }}$

$y=(\omega \cdot \rho \cdot L)(\sin \alpha \cdot \cos \phi+\cos \alpha \cdot \sin \phi)+y_{\text {ref }}$

Now the results obtained with $\rho=1, \omega=0.8$ and $\phi=10^{\circ}$ are shown in Fig. 9b.

Once the look-up tables are defined, as well as the equations to be used in the Hough transform, they can be implemented. The biggest problem we face is the fourdimensional search, which slows down the detection. Detection is improved by reducing the space of the search in the following manner:
- Parameter $\omega$ carries out the search between 0.90 and 1.20 , because if $\omega$ is reduced, the size of the ventricle would be too small and the doctor would not be able to find its characteristics. On the other hand, if $\omega$ is increased over 1.20, it would exceed the limits of the ultrasound.

- Parameter $\phi$ ranges between -300 and 300 , taking the $y$ axis as a reference. If we increase the degrees over 300 (or under -300 ) the ventricle would rotate so much that part of it would not be reflected in the echocardiography. A complete left ventricular long-axis view detection is attempted.

- Lastly, the maximum search space reduction is obtained by creating a window where reference point $\left(x_{\text {ref }}, y_{\text {ref }}\right)$ is more likely to be found. This is logical since half the image is not relevant in this study.

This window was obtained while trying to find the upper part of the ventricle. This is done by"looking" at the different intensity levels of the pixels which make up part of the gradient image. We begin in the upper part of the image and go down until we find a pixel that exceeds a certain threshold. Once this coordinate is obtained, the upper limit of the ventricle has been determined. This is done again to determine the lower, right and left limits. With these coordinates, the left ventricular boundary is defined. The following parameters can be calculated:

$$
\begin{aligned}
& C_{x}=\frac{x_{4}-x_{2}}{2}+x_{4} \\
& C_{y}=\frac{y_{3}-y_{1}}{2}+y_{1}
\end{aligned}
$$
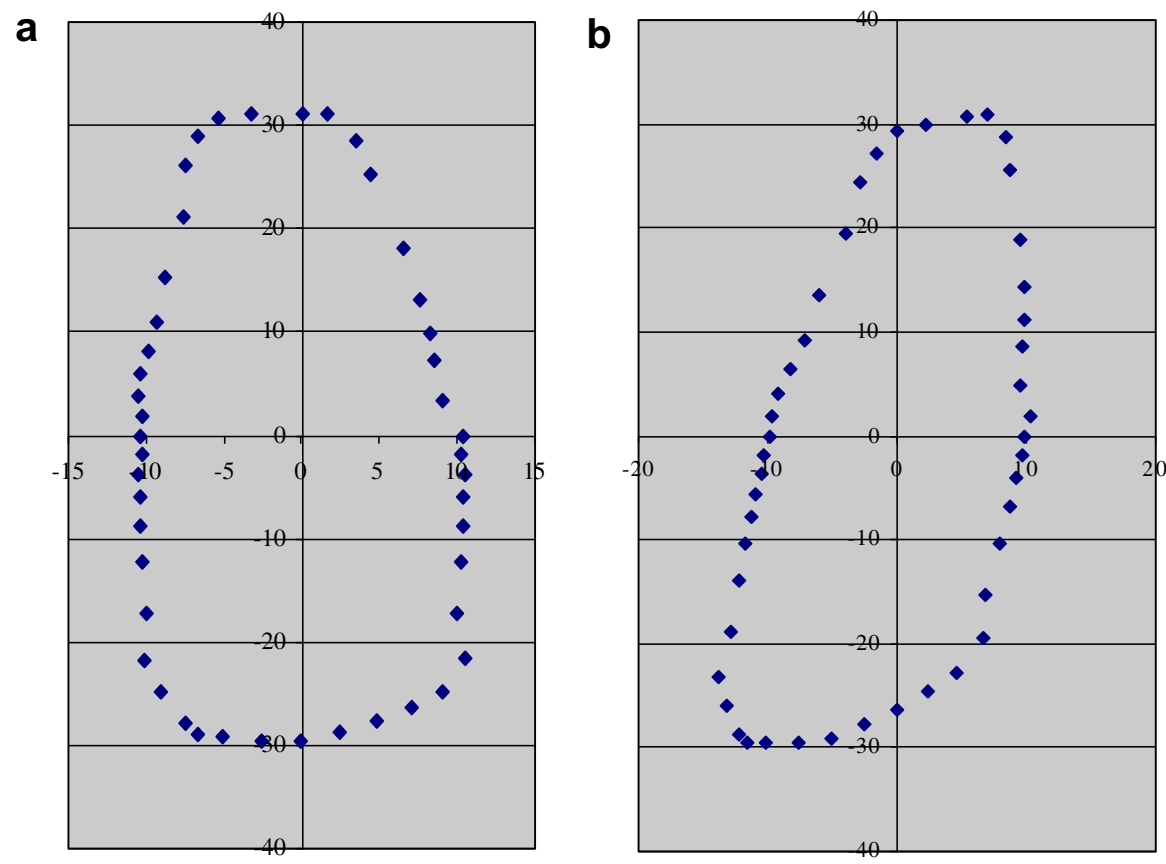

Fig. 9. (a) Representation after including the ventricle's size. (b) Representation after including the ventricle's rotation angle. 


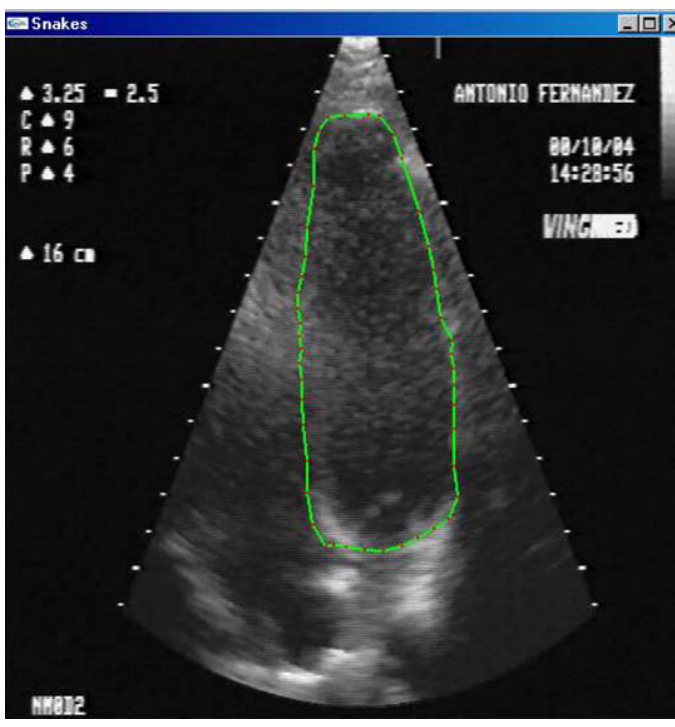

Fig. 10. Detection of the left ventricle (long-axis view).

which corresponds to the center of the ventricle $\left(C_{x}, C_{y}\right)$. With this parameter, we can define a window taking $\left(C_{x}, C_{y}\right)$ as its center.

Once the window has been defined, the search for the coordinate corresponding to the upper left corner will start. We check with the look-up table and the previous equations to find any coincidences, that is to say, if the coordinates that give us the equations exceed a certain threshold previously defined (in the smoothed image in operator DroG). If this is so, the accumulator increases by one. The said accumulator will be indexed by the reference point, by $\phi$ and by $\omega$. At the end of the search throughout the window, we will look for the accumulator's greatest value, which will indicate the long-axis left ventricular location. Fig. 10 shows a real image of the result of using the algorithm.

\subsection{Active-contours}

An active-contour or snake is a plain parametric contour model proposed by Kass et al. (1988) and made up of a set of characteristic pixels ( is controlled by the influence of a deformation or potential energy, which we try to minimize to reach a stability point. This model and its variations have been frequently used in the automatic follow-up of characteristics on biomedical images. The snake is a set of pixels which will be attracted by pixels of profiles in the image. A pixel in the image will attract more or less snaxels, depending on the energy associated to that pixel. The said energy is defined by the functions explained next. The classical formula for the global energy function is

$$
\begin{aligned}
E_{\text {total }}(v)= & \alpha(v) \int E_{\text {cont }}(v) \mathrm{d} v+\beta(v) \int E_{\text {curv }}(v) \mathrm{d} v \\
& +\gamma(v) \int E_{\text {image }}(v) \mathrm{d} v
\end{aligned}
$$

The said potential is made up of two types of energies of opposite signs: internal energy and external energy. The definition of the weighted terms that make up the global energy is the following one:

- Internal Energy: Defines the spatial relation between snaxels, as well as contour shape restrictions.

- Contour energy: This function forces snaxels $v_{i}$ to expand uniformly. The value indicates the mean distance between neighboring snaxels. The points in the image, where the snaxels would be more separated from the image of their nearest neighbors, attract the snaxel less. The energy comes from the absolute value of the mean distance between snaxels minus the distance between neighboring snaxels

$$
E_{\text {cont }}=\left|\bar{d}-\left\|v_{i}-v_{i-1}\right\|\right|
$$

- Curvature energy: This energy calculates the curvature at a point. We maintain so the shape restriction (global curvature)

$$
\begin{gathered}
E_{\text {curv }}=\left\|\frac{u_{i+1}}{\left\|u_{i+1}\right\|}-\frac{u_{i}}{\left\|u_{i}\right\|}\right\| \\
u_{i}=v_{i}-v_{i-1}
\end{gathered}
$$

- Image or external energy: This energy, of opposite signs to the previous one and also called external energy, is defined according to the characteristics we are trying to attract the snake towards (contours, high intensity points, distance transform). In the project model, it comes from the intensity value provided by the image smoothed with operator DroG.

All three energy terms must be in the same rank. In this case, they will be at the interval $[0,1]$. Parameters $\alpha$, $\beta$ and $\gamma$ are used to give more priority to one energy or another. We can handle the previous parameters according to the characteristics of the image or the processing we have to follow. The problem with snaxel minimization is formulated as a continuous minimization of this energy through subsequent potential changes. The said minimization can be approached in a voracious way through local minimization of the function in each snaxel's neighborhood.

\subsubsection{Mathematical description of the active-contours}

An active-contour is an orderly collection of $n$ points in the image plane:

$$
\begin{aligned}
V & =v_{i_{1}}, \ldots, v_{i_{n}} \\
v_{i} & =\left(x_{i}, y_{i}\right), \quad i=1 \ldots n
\end{aligned}
$$

The points in the contour approximate the limits of an object iteratively through a minimization problem. For every point in the $v_{i}$ neighborhood, the energy term is calculated:

$E_{i}=\alpha^{\prime} E_{\text {int }}\left(v_{i}\right)+\beta^{\prime} E_{\text {ext }}\left(v_{i}\right)$

where $E_{\text {int }}\left(v_{i}\right)$ is the energy function dependent on contour shape and $E_{\text {ext }}\left(v_{i}\right)$ is the energy function dependent on 
image properties, such as the gradient. $\alpha /$ and $\beta /$ are constant values that supply relative weight to the energy terms. $E_{i}, E_{\text {int }}$ and $E_{\text {ext }}$ are matrices. The central value for each matrix corresponds to the contour energy in point $v_{i}$. The rest of the values in the matrices corresponds to the energy in each point in the $v_{i}$ neighborhood. Each point $v_{i}$ is moved to point $v_{i}^{\prime}$, corresponding to the localization of the minimum value in $E_{i}$. If the energy functions are chosen correctly, contour $V$ should approximate and stop at the object boundary.

4.3.1.1. Internal energy. The internal energy function is geared at forcing a shape on the deformable contour and keeping a constant distance between contour pixels. Additional terms can be added to influence contour motion. The internal energy function can be defined as follows:

$\alpha^{\prime} E_{\text {int }}\left(v_{i}\right)=\alpha E_{\text {cont }}\left(v_{i}\right)+\beta E_{\text {curv }}\left(v_{i}\right)$

where $E_{\text {cont }}\left(v_{i}\right)$ is the continuity energy that forces the shape of the contour and $E_{\text {curv }}\left(v_{i}\right)$ is a curvature force that causes the contour to increase or to shrink. $\alpha$ and $\beta$ provide the relative weight of the energy terms.

4.3.1.2. Continuity or contour energy. In the absence of other influences, the continuity energy term forces an open deformable contour into a straight line and a closed deformable contour into a circle. The continuity energy formula is expressed next. The energy term for each element $e_{j k}\left(v_{i}\right)$ in matrix $E_{\text {cont }}\left(v_{i}\right)$ is defined as follows:

$e_{j k}\left(v_{i}\right)=\frac{1}{\tau(V)}\left\|P_{j k}\left(v_{i}\right)-\gamma\left(v_{i-1}-v_{i+1}\right)\right\|^{2}$

where $P_{j k}$ is the point in the image that corresponds spatially to the energy matrix element $e_{j k}\left(v_{i}\right) \cdot \gamma=0.5$ for open contours. In this case, the minimum energy point is the exact half-way point between $v_{i-1}$ and $v_{i+1}$. In the case of closed contours, $V$ is given by the modulus of $n$; therefore $v_{n+i}=v_{i} \cdot \gamma$ is defined as follows:

$\gamma=\frac{1}{2 \cos \frac{2 \pi}{n}}$

The normalization factor, $\tau(V)$, is the mean distance between every point in $V$ :

$\tau(V)=\frac{1}{n} \sum_{i=1}^{n}\left\|v_{i+1}-v_{i}\right\|^{2}$

4.3.1.3. Curvature energy or force. Curvature or balloon force is used on closed deformable contours to force the contour to expand (or shrink) in the absence of other external influences. A contour initialized within an object in a uniform image will expand under the influence of balloon force until it gets close to the object boundary. In Chalana, Haynor, and Kim (1994), an adaptable balloon force which will vary proportionally inversely to the image gradient magnitude is suggested. The adaptable balloon force is strong in homogenous regions and weak near object boundaries, edges and lines. The energy term for each element, $e_{j k}\left(v_{i}\right)$, in the matrix, $E_{\text {curv }}\left(v_{i}\right)$, is expressed as follows:

$e_{j k}\left(v_{i}\right)=n_{i} \cdot\left(v_{i}-p_{j k}\left(v_{i}\right)\right)$

where $n_{i}$ is the external unit normal for $V$ to point $v_{i}$ and $p_{j k}\left(v_{i}\right)$ are the neighboring points for $v_{i}$ corresponding to input $e_{j k}\left(v_{i}\right)$ in the energy matrix. Therefore, the balloon force is small at the points further away from $v_{i}$ in the direction of $n_{i} . n_{i}$ can be calculated by rotating the tangent vector $\left(t_{i}\right)$ :

$t_{i}=\frac{v_{i}-v_{i-1}}{\left\|v_{i}-v_{i-1}\right\|}+\frac{v_{i+1}-v_{i}}{\left\|v_{i+1}-v_{i}\right\|}$

Thus $n_{i}$ is a unit vector normal to $t_{i}$.

4.3.1.4. External energy. The external energy function attracts the deformable contour to more interesting characteristics, such as object boundaries in an image. Any expression that succeeds in doing this can be considered for use. Image gradient and intensity are obvious characteristics to be used (others could be object size or shape). Therefore, the external energy function could be the following one:

$\gamma E_{\text {ext }}\left(v_{i}\right)=m E_{\text {mag }}\left(v_{i}\right)$

where $m E_{\mathrm{mag}}\left(v_{i}\right)$ is an expression which attracts contours to high or low intensity regions. Moreover, constant $m$ is used to provide relative weight to the energy terms.

4.3.1.5. Image intensity energy. Each element in the intensity energy matrix, $m E_{\operatorname{mag}}\left(v_{i}\right)$ assigns the intensity values corresponding to the image points in the neighborhood of $v_{i}$ :

$e_{j k}\left(v_{i}\right)=I\left(p_{j k}\left(v_{i}\right)\right)$

If $m$ is positive, the contour is attracted to low intensity regions, but if $m$ is negative, it is attracted to high intensity regions. This depends on the scale being used. We can always move the scale towards positive values.

After having performed a Hough transform on the previous images and having found the left ventricular position in the short-axis as well as long-axis or two-chamber views, the next step is to execute the active-contour algorithm to perfect the solution by finding the exact shape of the different ventricular positions. Fig. 11 shows the result of applying the active-contour algorithm to both views of the left ventricle.

\subsection{Left ventricular length, area and wall thickness}

Once the active-contour has found the long-axis left ventricular view, its area, which corresponds to the area enclosed within the active-contour, is calculated. The following formula is used:

$2 S=\sum_{n=0} x_{n}\left(y_{n-1}-y_{n+1}\right)$ 

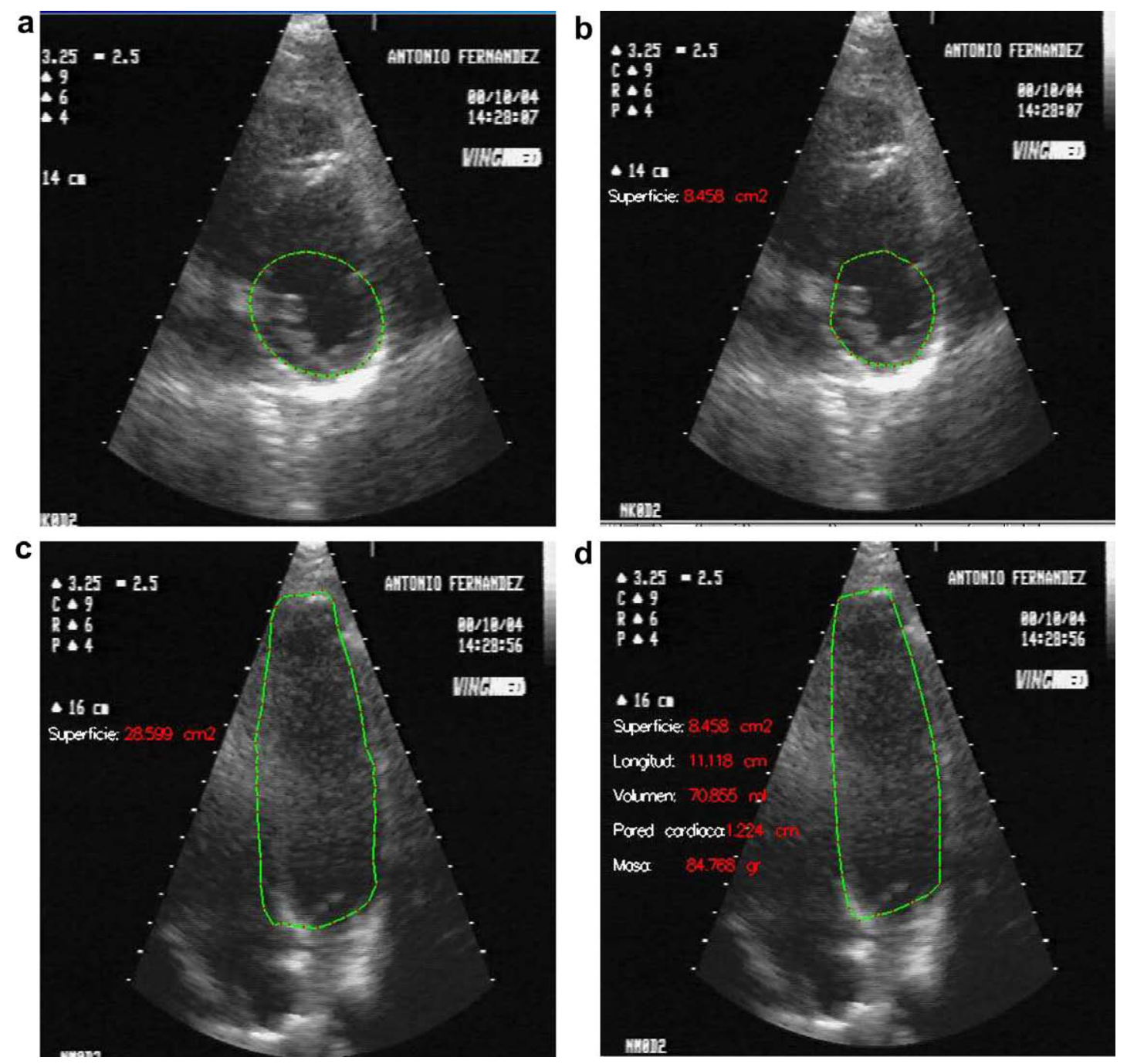

Fig. 11. (a) Detection of the left ventricle in the short-axis view. (b) Detection of the left ventricle in the short-axis view after active-contour algorithm. (c) Detection of the left ventricle in the long-axis view. (d) Detection of the left ventricle in the long-axis view after active-contour algorithm.

where $S$ represents the area, $x$ and $y$ represent the coordinates for the points which form the closed curve.

To calculate left ventricular length and thickness, it is necessary to have a long-axis view. Once the active-contour algorithm has been executed and the left ventricle has been detected, it is necessary to know both coordinates for those snaxels closest to the upper and lower parts of the image, respectively. Then, the length between both points is calculated. To calculate thickness, a point in the active-contour is taken and traced from that point towards the left and the right until it drops from a certain intensity threshold. The threshold defines the grey-level in the heart-wall. Once the extreme values are obtained, the length between those two points is calculated and thus ventricular thickness is obtained. This is done for five active-contour points and then the average length is calculated to obtain heart-wall thickness.

The results obtained may also be consulted in Fig. 11 . These are
- area (short-axis view): $8.458 \mathrm{~cm}^{2}$;

- length (long-axis view): $11.118 \mathrm{~cm}$;

- ventricular volume: $70.855 \mathrm{ml}$;

- heart-wall: $1.224 \mathrm{~cm}$;

- ventricular mass: $84.768 \mathrm{~g}$;

which fortunately correspond to a normal heart of one of the authors of the paper.

\section{Conclusions}

This article has described how to obtain a series of parameters from the heart's left ventricle for the diagnosis of certain heart disease. The bulk of this article has been dedicated to left ventricular segmentation in the shortand long-axis views to calculate cardiac parameters useful for physicians. Echocardiographic images have been used to support this. We have calculated parameters: (a) left ventricular area in a short-axis view, (b) left ventricular length in a long-axis view, (c) left ventricular volume, 
(d) left ventricular mass, and (e) heart-wall thickness, which allow us to detect if the patient being studied suffers from cardiopathy.

For these reasons, this article describes different algorithms for left ventricular detection in two-dimensional echocardiographic images, in short-axis as well as long-axis views. Once the ventricle has been detected, a series of parameters, which define that ventricle, have been calculated.

The highlight of this proposal is that it is not necessary to select the points or to color the contour manually to detect it (as it is done in other methods), but that it can be done automatically. All this is based on two types of algorithms: active-contours and Hough transforms. A Hough transform detects the ventricle's shape in two views. Once the first objective has been achieved, the points obtained by the transform are transferred to the active-contour algorithm, which then approximates the curve to the ventricle. This automatism prevents the mistakes made with manual detection. Let us remember once more that this is one of the problems physicians face when making a diagnosis using echocardiographic devices. Thanks to this new implementation, we can avoid the observer's uncertainty.

As usual, there are always improvements to be made to the system described. For example, the algorithm used for active-contour energy minimization is a greedy algorithm. The results could be improved if a different type of algorithm were used. Other possible improvements are pre-processing the images through more sophisticated filters, eliminating noisy edges more efficiently, defining the edges more accurately and/or speeding up the detection.

Another possible improvement in the detection software would be to incorporate information about the stage of ventricular dilation. That is to say, if it is in systole, diastole or in-between. This is fairly easy to calculate by considering ventricular area. Ejection force could also be calculated this way.

\section{References}

Angelini, E. D., Homma, S., Pearson, G., Holmes, J. W., \& Laine, A. F. (2005). Segmentation of real-time three-dimensional ultrasound for quantification of ventricular function: A clinical study on right and left ventricles. Ultrasound in Medicine \& Biology, 31, 1143-1158.

Braunwald, E., Zipes, D. P., Libby, P., \& Bonow, R. (2004). Braunwald's Heart Disease: A Textbook of Cardiovascular Medicine. W.B. Saunders Co.
Buda, A. J., Delp, E. J., Meyer, C. R., Jenkins, J. M., Smith, D. N., Booktein, F. L., et al. (1983). Automatic computer processing of digital 2-dimensional echocardiograms. The American Journal of Cardiology, 52, 384-389.

Chalana, V., Haynor, D. R., \& Kim, Y. (1994). Left-ventricular boundary detection from short-axis echocardiograms: The use of active contour models. SPIE Conference on Medical Imaging, 2167, 786-798.

Chalana, V., Linker, D. T., Haynor, D. R., \& Kim, Y. (1996). A multiple active contour model for cardiac boundary detection on echocardiographic sequences. IEEE Transactions on Medical Imaging, 15, 290-298.

Chu, C. H., Delp, E. J., \& Buda, A. J. (1988). Detecting left ventricular endocardial and epicardial boundaries by digital two dimensional echocardiography. IEEE Transactions on Medical Imaging, 7, 81-90.

Fuster, V., Alexander, R. W., \& O'Rourke, R. A. (2001). Hurst's the Heart. McGraw-Hill.

Kass, M., Witkin, A., \& Terzopoulos, D. (1988). Snakes: Active contour models. International Journal of Computer Vision, 1, 321-331.

Malassiotis, S., \& Strintzis, M. G. (1999). Tracking the left ventricle in echocardiographic images by learning heart dynamics. IEEE Transactions on Medical Imaging, 18, 282-290.

Mikic, I., Krucinski, S., \& Thomas, J. D. (1998). Segmentation and tracking in echocardiographic sequences: Active contours guided by optical flow estimates. IEEE Transactions on Medical Imaging, 17, 274-284.

Mishra, A., Dutta, P. K., \& Ghosh, M. K. (2003). A GA based approach for boundary detection of left ventricle with echocardiographic image sequences. Image and Vision Computing, 21, 967-976.

Montagnat, J., Sermesant, M., Delingette, H., Malandain, G., \& Ayache, N. (2003). Anisotropic filtering for model-based segmentation of 4D cylindrical echocardiographic images. Pattern Recognition Letters, 24, $815-828$.

Nastar, A., \& Ayache, N. (1993). Fast segmentation, tracking, and analysis of deformable objects. In Fourth international conference on computer vision, ICCV'93 (pp. 275-279).

Paragios, N., Jolly, M. P., Taron, M., \& Ramaraj, R. (2005). Active shape models \& segmentation of the left ventricle in echocardiography. Lecture Notes in Computer Science, 3459, 131-142.

Pardo, X. M., Solé, A. F., \& Cabello, D. (2000). Active segmentation of biomedical images: Edge saliency and region dissimilarity. Pattern Recognition and Applications, 56, 233-242.

Pincum, M., Schwartz, G., Corday, S. R., Fujibayashi, Y., \& Meerbaum, S. (1986). Attenuation correction in echocardiography. Ultrasonic Imaging, 8, 86-106.

Ranganath, S. (1995). Contour extraction from cardiac MRI studies using snakes. IEEE Transactions on Medical Imaging, 14, 328-338.

Shekhar, R., Zagrodsky, V., \& Walimbe, V. (2004). 3D Stress echocardiography: Development of novel visualization, registration and segmentation algorithms. International Congress Series, 1268 , 1072-1077.

Staib, L. H., \& Duncan, J. S. (1992). Boundary finding with parametrically deformable models. IEEE Transactions on Pattern Analysis and Machine Intelligence, 14, 1061-1074.

Yan, H., Jiang, Y., Zheng, J., Peng, C., \& Li, Q. (2006). A multilayer perceptron-based medical decision support system for heart disease diagnosis. Expert Systems with Applications, 30, 272-281. 